\title{
Concentration dependence of energy transfer upconversion in Nd:YAG
}

\author{
S. J. Yoon ${ }^{1}$, R. P. Yan ${ }^{1,2}$, S. J. Beecher ${ }^{1}$, J. I. Mackenzie ${ }^{1}$ \\ ${ }^{1}$ Optoelectronics Research Centre, University of Southampton, Southampton SO17 1BJ, United Kingdom; \\ ${ }^{2}$ National Key Laboratory of Science and Technology on Tunable Laser, Harbin Institute of Technology, Harbin 150080, China \\ Author e-mail address: sjy1g12@orc.soton.ac.uk
}

\begin{abstract}
The concentration dependence of energy transfer upconversion in Nd:YAG is investigated via the $\mathrm{z}$-scan technique. The ETU coefficient is determined to increase from $35 \times 10^{-18} \mathrm{~cm}^{3} / \mathrm{s}$ to $90 \times 10^{-18} \mathrm{~cm}^{3} / \mathrm{s}$ when the concentration increases from 0.3 at. $\%$ to 1.1 at. $\%$.

OCIS codes: (190.7220) Upconversion; (140.3530) Lasers, neodymium; (300.6460) Spectroscopy, saturation.
\end{abstract}

\section{Introduction}

Since the inception of solid-state lasers, the neodymium doped crystals have been widely used to generate lasers in near-infrared region. Due to its excellent spectroscopic and thermo-optic properties, Nd:YAG, is one of the prime active crystals for many industrial, medical and scientific laser systems. Primarily it is employed in lasers operating on the dominant emission line around $1.06 \mu \mathrm{m}$, however, the lower gain $0.9 \mu \mathrm{m}$ transition potentially could be more efficient due to a lower quantum defect between pump and output wavelengths. Unfortunately this latter transition is weak and susceptible to detrimental thermal effects associated with waste heat during the excitation process. Many approaches have been employed to dissipate the heat-load efficiently and mitigate the ensuing thermal effects via the laser architecture, essentially the geometry of the gain medium, with the output powers at $946 \mathrm{~nm}$ now exceeding $100 \mathrm{~W}$ [1]. However, despite the maturity of this gain material further improvement in its laser performance may be possible if key fundamental contributors to the thermal input are well characterized. For this purpose we have configured a simple experimental setup to characterize one such parameter, namely energy transfer upconverion (ETU) and its dependence upon the neodymium concentration.

During the ETU process, two neighboring ions in their meta-stable $\left({ }^{4} \mathrm{~F}_{3 / 2}\right)$ energy state interact, one of which relaxes to a lower level while the other one is excited it to a higher energy level. Therefore ETU depopulates the upper laser level, generating additional heat via the non-radiative relaxation paths taken by both ions, the first arriving in its ground state, while for the majority of these pairs the second returns back to the meta-stable manifold that both were in originally. The influence of ETU can be quite significant for the laser design and optimization, especially for low gain lasers that typically require high-irradiance pumping, such as the quasi-four-level $\mathrm{Nd}^{3+}$ system. It is relatively well known that the magnitude of ETU coefficient is dependent on the concentration of the rare earth ion, and is an important parameter that is currently not well covered in the literature for Nd:YAG.

In this work, we investigated the concentration dependence of the ETU coefficient in Nd:YAG with a sensitive zscan technique, comparing measured transmission as a function of incident pump irradiance with simulation based upon a two-level spatially (and temporally) dependent rate equation system, with the only variable being the ETU coefficient (once a cross relaxation parameter is fixed for the concentration in question). The ETU coefficient was found to increase from $35 \pm 5 \times 10^{-18} \mathrm{~cm}^{3} / \mathrm{s}$ to to $90 \pm 10 \times 10^{-18} \mathrm{~cm}^{3} / \mathrm{s}$ when the doping concentration increased from 0.3 at $\%$ to 1.1 at $\%$.

\section{Methodology}

The z-scan technique is a simple method, in which the change in the transmission of a pump laser through the sample is measured as the crystal is moved through a focus, which is then correlated to the saturation irradiance. ETU weakens the ground state bleaching, effectively reducing the expected amplitude of the transmission at the point of highest pump irradiance. This process provides a surprisingly sensitive measure of the magnitude of the ETU coefficient. The detail of our theoretical model can be found in our previous paper [2].

A schematic of the $\mathrm{z}$ scan experiment setup is shown in Fig 1. The output beam of continuous wave Ti:Sapphire laser was expanded 4 times with a telescope system and modulated mechanically with a chopper. The pulse duration of $\sim 2.4 \mathrm{~ms}$ is long enough to reach steady state, while the duty cycle of $10 \%$ is small enough to eliminate the influence of additional heat generated in sample. A focusing lens of $f=200 \mathrm{~mm}$ was mounted to an electronicallycontrolled translation stage (Stackshot, Cognisys Inc.) to change the beam size in the laser crystal, which can provide a precise control the irradiance in the sample. The transmitted power was collimated and split with a wedge, of which $\sim 92 \%$ passes directly to a power meter (Up19K, Gentec-EO), while one surface reflection ( 4\%) was 
redirected to a silicon photodiode. With a concave mirror (L5) and a focusing lens (L6), the fluorescence was collected and delivered to an InGaAs photodiode. A digital oscilloscope (MSO6104A, Agilent Technology Inc.) could be used to record the amplitude of transmitted signal and measure the fluorescence lifetime. Using a beam profiler (Nanoscan II, OPHIR Inc.) placed at the position of sample, the laser beam size along z-axis was measured.

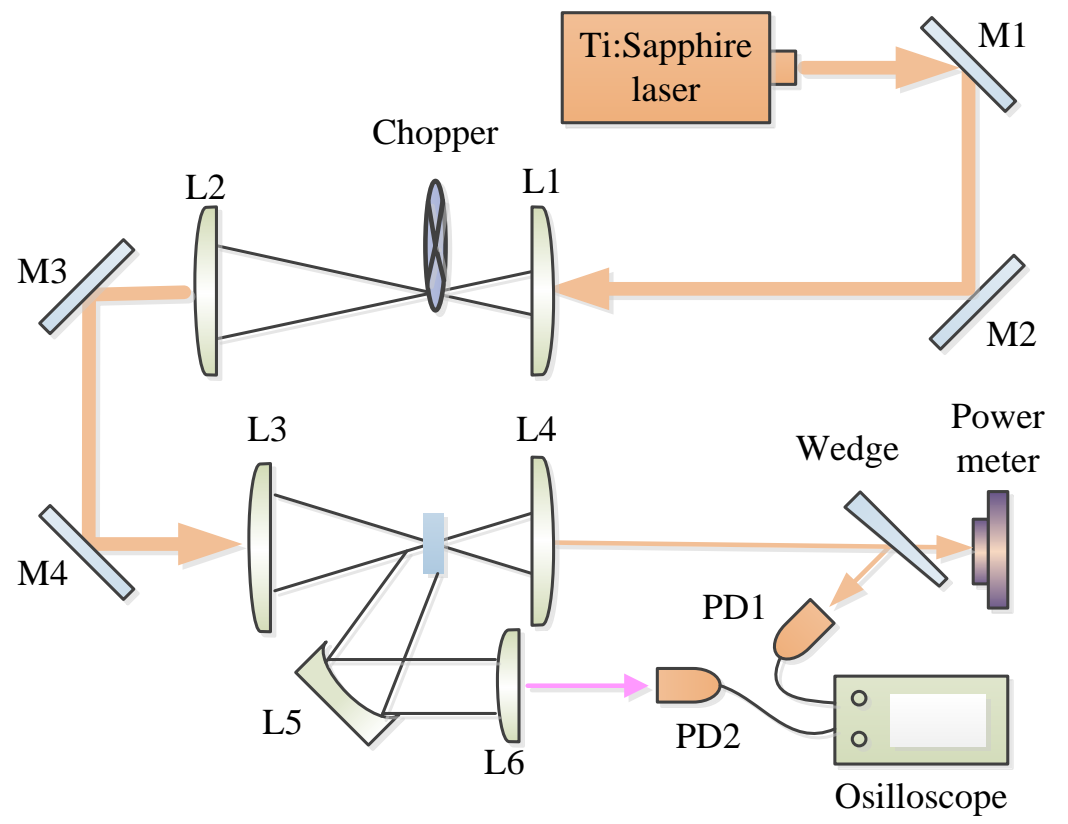

Fig 1. Experimental setup: M1, M2, M3, M4: broad band reflecting mirror; L1: $f=75 \mathrm{~mm}$ lens, L2: $f=300 \mathrm{~mm}$ lens, L3: $f=200 \mathrm{~mm}$ lens, L4: $f=$ $100 \mathrm{~mm}$ lens, L5: $f=-50 \mathrm{~mm}$ concave mirror, L6: $f=50 \mathrm{~mm}$ lens, PD1: high speed Si photodiode (Thorlabs, DET210), PD2: high speed InGaAs photodiode (Thorlabs, DET410).

\section{Experimental results and discussion}

Table 1. The parameters of Nd:YAG sample

\begin{tabular}{|c|c|c|c|c|}
\hline Sample & 1 & 2 & 3 & 4 \\
\hline Doping Concentration (at.\%) & 1.1 & 1.0 & 0.6 & 0.3 \\
\hline Length (mm) & 1.1 & 3.2 & 1.1 & 5.1 \\
\hline ETU $\left({\left.\mathrm{x} 10^{-18} \mathrm{~cm}^{3} / \mathrm{s}\right)}\right)$ & 90 & 65 & 35 & 35 \\
\hline
\end{tabular}

The beam quality of the pump laser beam was measured to be $M^{2}=1$ in both directions, providing beam waist radii of $\omega_{x}=20.7 \pm 0.2 \mu \mathrm{m}$ and $\omega_{y}=19.7 \pm 0.2 \mu \mathrm{m}$ after the focusing lens of $f=200 \mathrm{~mm}$. The "on axis" available pump irradiance reached $67 \mathrm{kWcm}^{-2}$, nearly five times higher than the saturation irradiance, when the wavelength was tuned to the absorption peak of Nd:YAG around $808.5 \mathrm{~nm}$. The ETU parameter measured at weaker irradiance levels was found to be almost identical, if the irradiance was higher than the crystal's saturation irradiance. Higher irradiance was required to verify the curve fit between measured and simulated transmission. Four Nd:YAG samples have been tested, as shown in Table 1, to determine the variation of the ETU coefficient as a function of the neodymium concentration. The fluorescence lifetime of Nd:YAG of the 1.0 at.\% crystal was also measured and in the small signal regime the major component was found to be $235+/-5 \mu$ s as is typical for this concentraion. In the high irradiance regime, this value reduced to $220+/-5 \mu \mathrm{s}$, and we will discuss the comparison of the ETU coefficient derived from this value in comparison to that obtained from measurement of the transmitted power. For the $1.1 \%$ doped crystal, a longer crystal was also measured to compare it with a shorter crystal to ensure there was no dependence on the crystal length, for which we measured an ETU coefficient within error range for each.

Table 2. The fluorescence lifetime for different doping concentrations

\begin{tabular}{|c|c|c|c|c|}
\hline Doping Concentration (at.\%) & 1.1 & 1.0 & 0.6 & 0.3 \\
\hline Fluorescence lifetime with weak excitation $(\mu \mathrm{s})$ & 232 & 235 & 250 & 252 \\
\hline $\mathrm{W}_{\mathrm{cr}}\left(\mathrm{x} 10^{-18} \mathrm{~cm}^{3} / \mathrm{s}\right)$ & 3.06 & 2.96 & 1.85 & 2.94 \\
\hline
\end{tabular}

We used $\mathrm{W}_{\mathrm{cr}}$ values determined from the fluorescence lifetime taken with weak excitation, assuming that the intrinsic lifetime of $\mathrm{Nd}^{3+}$ in YAG is $260 \mu$ s [3], and under very low pump excitation this is the only decay mechanism apart from spontaneous emission, giving a value of $2.96 \times 10^{-18} \mathrm{~cm}^{3} / \mathrm{s}$ for $1 \%$ doped crystal. Using the 
data of cross relaxation coefficients, the dependence of ETU coefficient on different doping concentrations is obtained from fitting of the transmission as a function of the crystal position (z-scan position) and the final values shown in Fig. 2. The ETU coefficients are $35 \pm 5 \times 10^{-18} \mathrm{~cm}^{3} / \mathrm{s}$ for 0.3 at. $\%$ doped Nd:YAG increasing to $90 \pm 10 \times 10^{-18} \mathrm{~cm}^{3} / \mathrm{s}$ at the doping concentration of $1.1 \mathrm{at} . \%$. It can be seen that the ETU coefficient increases with doping concentration rising, consistent with previous results in neodymium doped materials [4]. This is expected as ETU is strongly dependent upon the distance between neighbouring ions, which is reducing at higher doping levels. For some of the samples investigated there appeared to be a strong transverse variation in the transmission, assumed to be a non-uniform distribution of the $\mathrm{Nd}^{3+}$, and which was found to reduce the accuracy of the experimental results.
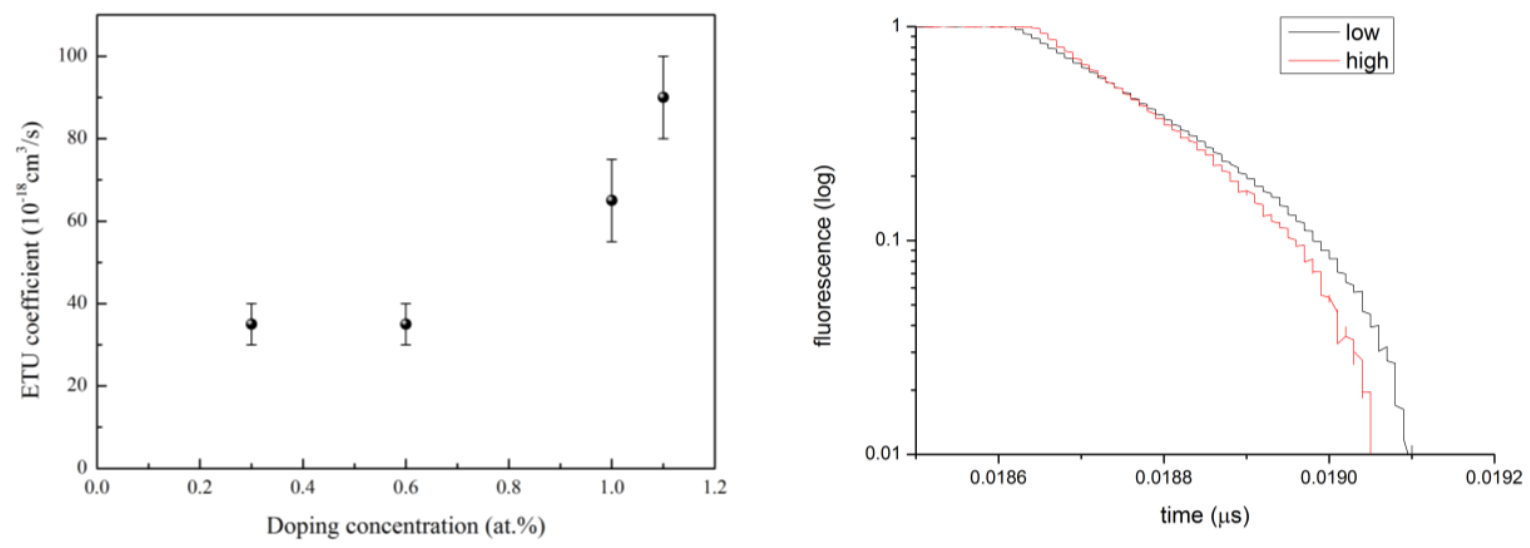

Fig. 2 The concentration dependence of ETU coefficients for Nd:YAG and fluorescence lifetime

\section{Conclusions}

In conclusion, we have determined the variation of ETU coefficient for different doping concentration in $\mathrm{Nd}$ :YAG crystal via the z-scan technique. This method gives us a precise measurement of transmission change with pump irradiance when the sample is moved along the beam path. Using a two-level rate equation, the experiment data is well matched by fitting only the ETU coefficient. We obtained values of $35 \pm 5 \times 10^{-18} \mathrm{~cm}^{3} / \mathrm{s}$ to $90 \pm 10 \times 10^{-18} \mathrm{~cm}^{3} / \mathrm{s}$ when the doping concentration increases from 0.3 at $\%$ to 1.1 at $\%$. Further investigation is currently underway to measure the ETU coeffcient in other materials, mainly neodymium doped vanadates.

\section{References}

[1] S. P.Ng, and J. I.Mackenzie, "Power and Radiance Scaling of a 946 nm Nd:YAG Planar Waveguide Laser," Laser Phys. 22, 494-498 (2012).

[2] R. Yan, S. Yoon, S. Beecher, J. Mackenzie, “Measurement of energy transfer upconversion in Nd:YAG via the z-scan technique,” Proc. SPIE 9135, Laser Sources and Applications II, 913525

[3] V. Lupei and A. Lupei, "Emission dynamics of the ${ }^{4} \mathrm{~F}_{3 / 2}$ level of $\mathrm{Nd}^{3+}$ in $\mathrm{YAG}$ at low pump intensities," Phys. Rev. B, vol. 61, no. 12, pp. 8087-8098, Mar. 2000.

[4] S. Guy, C. L. Bonner, D. P .Shepherd, D. C. Hanna, A. C. Tropper, B. Ferrand, "High-Inversion Densities in Nd:YAG: Upconversion and Bleaching," IEEE J. Quantum Electron. 34, 900-909 (1998). 